\title{
Psicooncología
}

ISSN: $1696-7240$

\section{El panorama internacional de la psico-oncología en la actualidad}

\author{
Maria Die Trill ${ }^{1}$
}

El pasado mes de Agosto se celebró en Berlín el decimonono congreso de la International Psycho-Oncology Society - IPOS. A pesar de la interrupción vacacional que supuso la asistencia al congreso para muchos profesionales dedicados a la psicooncología, la audiencia superó los 700 asistentes.

La misión de la IPOS es promocionar la excelencia en el cuidado psicosocial de personas afectadas por cáncer a nivel global a través de acuerdos, investigación, alianzas, participación en política sanitaria y formación. El lema del congreso era CANCER THROUGHOUT THE LIFESPAN: ADDRESSING THE PSYCHOSOCIAL NEEDS OF DIVERSE POPULATIONS. Se ha hecho un énfasis especial en la carga que supone padecer ciertos síntomas así como en el distress y en las necesidades psicosociales a lo largo de las diferentes etapas evolutivas desde la infancia y adolescencia hasta la vejez, así como en las necesidades de diferentes poblaciones oncológicas.

Durante el congreso se ha hecho especial énfasis en apoyar a los jóvenes profesionales que comienzan sus carreras y que constituyen uno de los grupos más activos y mejor representados en IPOS. Para ellos se han impartido talleres sobre el diseño de proyectos de investigación y sesiones de posters específicos además de tutorías. Como novedad se han incluido las llamadas "oncology educationals", que consisten en simposia que versan sobre los nuevos desarrollos en oncología y novedades en el cuidado al paciente (oncología para psicólogos). Estos incluyeron sesiones sobre la Oncología Personalizada y su Impacto en el Cuidado del Paciente, Salud Sexual e Infertilidad, y Beneficios del Tratamiento y Manejo de Efectos Secundarios de Terapias Noveles en Oncología.

De interés especial fue la sesión Plenaria del Presidente que este año trató el tema: IMPLEMENTANDO INNOVACIÓN PSICOSOCIAL EN EL TRATAMIENTO DEL CÁNCER: CÓMO PODEMOS FACILITAR EL CAMBIO, en la que participaron personajes muy representativos de la oncología mundial: Alberto Costa, Presidente de la Escuela Europea de Oncología; Andreas Ullrich, representante de la Organización Mundial de la Salud con quien IPOS colabora y es miembro desde hace aproximadamente 10 años; Barry Bultz, Presidente Pasado de IPOS; Paolo Casali, representante de ESMO (European Society of Medical Oncology); Peter Naredi, Presidente de la ECCO (European Cancer Organizations); Tit Albreht, Coordinador de la EPAAC y CANCON; e Isabelle Manneh-Vangramberen de la ECPC (European Cancer Patients Coalition). La sesión fue moderada por Luzía Travado, y Maria Die

Presidenta, International Psycho-Oncology Society.

Dirección de correspondencia. María Die Trilla. Atrio, c/ del Cinca, 25, 28002 Madrid. E-mail: mdietrill@gmail.com 
Trill comentó las ponencias y cerró la sesión. El objetivo de ésta fue que cada uno de los ponentes identificara maneras en las que las instituciones que representaban podrían apoyar la misión de IPOS de introducir la atención psicosocial como parte integral y mandatoria del cuidado. Facilitar la formación de los profesionales sanitarios en cuestiones psicosociales con el fin de gradualmente producir cambios en la actitud hacia la psicooncología fue una de las tareas identificadas. Una de las consecuencias que ha surgido de nuestras colaboraciones con estos organismos ha sido la inclusión por primera vez, de un simposium conjunto IPOS - ESMO sobre el burnout en el congreso recientemente celebrado en Madrid de la Sociedad Europea de Oncología Médica ESMO al que acudieron 23.000 asistentes.

Predominaron en IPOS Berlín sesiones centradas en la supervivencia del cáncer, las habilidades de comunicación, intervención psicosocial en cánceres avanzados, prevención, cuidados paliativos, e intervenciones psicológicas basadas en la evidencia.

Por mencionar queda mi nombramiento tras una votación entre los miembros de IPOS, como Presidenta de la sociedad. Es todo un honor para mi poder representar la psicooncología a nivel mundial, tarea que he comenzado a hacer creando un grupo de trabajo dentro de IPOS llamado LATIN AMERICAN AND SPANISH SPECIAL INTEREST GROUP (LASSIG) en el que ya hay cerca de 50 inscritos y que va a servir para aumentar la presencia y participación de hispanoparlantes y portugueses en la sociedad. Se han creado grupos de trabajo dentro de LASSIG que van a facilitar la colaboración y desarrollo de proyectos entre profesionales de diferentes países. Para formar parte del grupo es necesario ser miembro de IPOS y contactar directamente conmigo. Además, estoy trabajando en conseguir apoyo de la OMS para extender la psicooncologia en Latino-America, así como en fomentar la participación de grupos de pacientes, enfermeras y profesionales de los cuidados paliativos en IPOS.

La celebración de los congresos anuales de IPOS constituye la mayor oportunidad científica para discutir los nuevos insights y planificar nuevos proyectos en psicooncología. El congreso ofrece una oportunidad inspiradora para el intercambio de conocimientos entre científicos, clínicos, políticos y todos aquellos implicados en la especialidad de la psicooncologia. Sirve además para afianzar la misión de IPOS de considerar la atención psicosocial como derecho de cualquier paciente con cáncer. Por ello os invito a que participéis de forma activa en IPOS aprovechando la presidencia Española (¡!), la formación de LASSIG, y la oportunidad de viajar a Hong Kong en 2018 y a Canadá en 2019, próximas sedes del congreso.

Espero vuestras noticias, vuestras ideas y vuestra participación en una sociedad que debemos conseguir sea más representativa de nuestros grupos de trabajo.

¡Muchas gracias por vuestro apoyo! 